\title{
A prospective study of dietary acrylamide intake and the risk of endometrial, ovarian, and breast cancer
}

Citation for published version (APA):

Hogervorst, J. G., Schouten, L. J., Konings, E. J., Goldbohm, R. A., \& van den Brandt, P. A. (2007). A prospective study of dietary acrylamide intake and the risk of endometrial, ovarian, and breast cancer. Cancer Epidemiology Biomarkers \& Prevention, 16(11), 2304-2313. https://doi.org/10.1158/10559965.EPI-07-0581

Document status and date:

Published: 01/01/2007

DOI:

10.1158/1055-9965.EPI-07-0581

Document Version:

Publisher's PDF, also known as Version of record

\section{Please check the document version of this publication:}

- A submitted manuscript is the version of the article upon submission and before peer-review. There can be important differences between the submitted version and the official published version of record.

People interested in the research are advised to contact the author for the final version of the publication, or visit the DOI to the publisher's website.

- The final author version and the galley proof are versions of the publication after peer review.

- The final published version features the final layout of the paper including the volume, issue and page numbers.

Link to publication

\footnotetext{
General rights rights.

- You may freely distribute the URL identifying the publication in the public portal. please follow below link for the End User Agreement:

www.umlib.nl/taverne-license

Take down policy

If you believe that this document breaches copyright please contact us at:

repository@maastrichtuniversity.nl

providing details and we will investigate your claim.
}

Copyright and moral rights for the publications made accessible in the public portal are retained by the authors and/or other copyright owners and it is a condition of accessing publications that users recognise and abide by the legal requirements associated with these

- Users may download and print one copy of any publication from the public portal for the purpose of private study or research.

- You may not further distribute the material or use it for any profit-making activity or commercial gain

If the publication is distributed under the terms of Article $25 \mathrm{fa}$ of the Dutch Copyright Act, indicated by the "Taverne" license above, 


\title{
A Prospective Study of Dietary Acrylamide Intake and the Risk of Endometrial, Ovarian, and Breast Cancer
}

\author{
Janneke G. Hogervorst, ${ }^{1}$ Leo J. Schouten, ${ }^{1}$ Erik J. Konings, ${ }^{2}$ R. Alexandra Goldbohm, ${ }^{3}$ \\ and Piet A. van den Brandt $\mathbf{1}^{1}$ \\ ${ }^{1}$ Department of Epidemiology, Maastricht University, Maastricht, the Netherlands; ${ }^{2}$ Food and Consumer Product Safety Authority, \\ Region South, Department Research \& Development, Eindhoven, the Netherlands; and ${ }^{3}$ Department of Food and Chemical Risk \\ Analysis, TNO Quality of Life, Zeist, the Netherlands
}

\begin{abstract}
Background: Acrylamide, a probable human carcinogen, was detected in various heat-treated carbohydrate-rich foods in 2002. The few epidemiologic studies done thus far have not shown a relationship with cancer. Our aim was to investigate the association between acrylamide intake and endometrial, ovarian, and breast cancer risk. Methods: The Netherlands Cohort Study on diet and cancer includes 62,573 women, aged 55-69 years. At baseline (1986), a random subcohort of 2,589 women was selected using a case cohort analysis approach for analysis. The acrylamide intake of subcohort members and cases was assessed with a food frequency questionnaire and was based on chemical analysis of all relevant Dutch foods. Subgroup analyses were done for never-smokers to eliminate the influence of smoking; an important source of acrylamide.

Results: After 11.3 years of follow-up, 327, 300, and 1,835 cases of endometrial, ovarian, and breast cancer,

respectively, were documented. Compared with the lowest quintile of acrylamide intake (mean intake, $8.9 \mu \mathrm{g} /$ day), multivariable-adjusted hazard rate ratios (HR) for endometrial, ovarian, and breast cancer in the highest quintile (mean intake, $40.2 \mu \mathrm{g} /$ day) were $1.29[95 \%$ confidence interval $(95 \% \mathrm{CI}), \quad 0.81-2.07$; $\left.P_{\text {trend }}=0.18\right], 1.78\left(95 \% \mathrm{CI}, 1.10-2.88 ; P_{\text {trend }}=0.02\right)$, and $0.93\left(95 \% \mathrm{CI}, 0.73-1.19 ; P_{\text {trend }}=0.79\right)$, respectively. For never-smokers, the corresponding HRs were 1.99 (95\% CI, 1.12-3.52; $\left.P_{\text {trend }}=0.03\right), 2.22$ (95\% CI, $1.20-$ $\left.4.08 ; P_{\text {trend }}=0.01\right)$, and $1.10(95 \%$ CI, 0.80-1.52; $\left.P_{\text {trend }}=0.55\right)$.

Conclusions: We observed increased risks of postmenopausal endometrial and ovarian cancer with increasing dietary acrylamide intake, particularly among never-smokers. Risk of breast cancer was not associated with acrylamide intake. (Cancer Epidemiol Biomarkers Prev 2007;16(11):2304-13)
\end{abstract}

\section{Introduction}

In 2002, Swedish researchers reported the presence of acrylamide, classified by the IARC as a probable human carcinogen, in several heat-treated carbohydrate-rich foods (1). This message received a lot of media attention and caused concern among the general population and the scientific community, particularly because acrylamide is present in foods at considerably higher levels than other well-known food carcinogens, such as polycyclic aromatic hydrocarbons and ethyl carbamate (2). Before this date, acrylamide exposure was thought to occur mainly through occupational exposure because it has been widely used in industry since the 1950s, and further through cigarette smoke and, to a minor extent, through drinking water $(3,4)$. Since 2002, research in several countries has also shown high acrylamide levels in foods such as French fries, coffee, potato crisps, and cookies (4). In the Netherlands, high levels of acrylamide were found

Received 6/27/07; revised 8/14/07; accepted 9/5/07.

Grant support: Dutch Food and Consumer Product Safety Authority (VWA). The NLCS was established with funding from the Dutch Cancer Society (KWF). The costs of publication of this article were defrayed in part by the payment of page charges. This article must therefore be hereby marked advertisement in accordance charges. This article must therefore be hereby marked
with 18 U.S.C. Section 1734 solely to indicate this fact.

Requests for reprints: Janneke Hogervorst, Department of Epidemiology, Maastricht University, P.O. Box 616, 6200 MD, Maastricht, the Netherlands. Phone: 31-433882391; Fax: 31-433884128. E-mail: JGF.Hogervorst@EPID.Unimaas.nl

Copyright (C) 2007 American Association for Cancer Research.

doi:10.1158/1055-9965.EPI-07-0581

in the typically Dutch spiced cakes (5), which are often consumed daily.

Acrylamide has been shown to be mainly formed in food by Maillard browning reactions in which amino acids, mainly asparagine, react with reducing sugars at high temperatures $\left(>120^{\circ} \mathrm{C}\right.$; refs. 6-8).

It is generally thought that acrylamide might cause cancer through cytochrome P4502E1 (CYP2E1)-catalyzed conversion to glycidamide, which has proven to be genotoxic (4).

There is a lot of debate on whether acrylamide in food justifies the concern that it provoked in 2002. Animal studies have shown positive dose-response relationships between acrylamide given in drinking water and cancer in multiple organs in both mice and rats (9-12), among which were several hormone-sensitive organs, such as the mammary glands and the uterus. However, studies on humans with occupational acrylamide exposure have been negative thus far, apart from an increased risk of pancreatic cancer, but this was based on a small number of cases (13-18). Dietary acrylamide intake and its relation with various types of cancer have been studied in a few case-control studies (19-21) and only two prospective cohort studies on breast and colorectal cancer $(22,23)$. None of these have found indications for a positive relationship.

Because of the pitfalls of case-control studies and the paucity of cohort studies, there is a need of more 
Figure 1. Flow diagram of subcohort members and cases on whom the analyses were based.

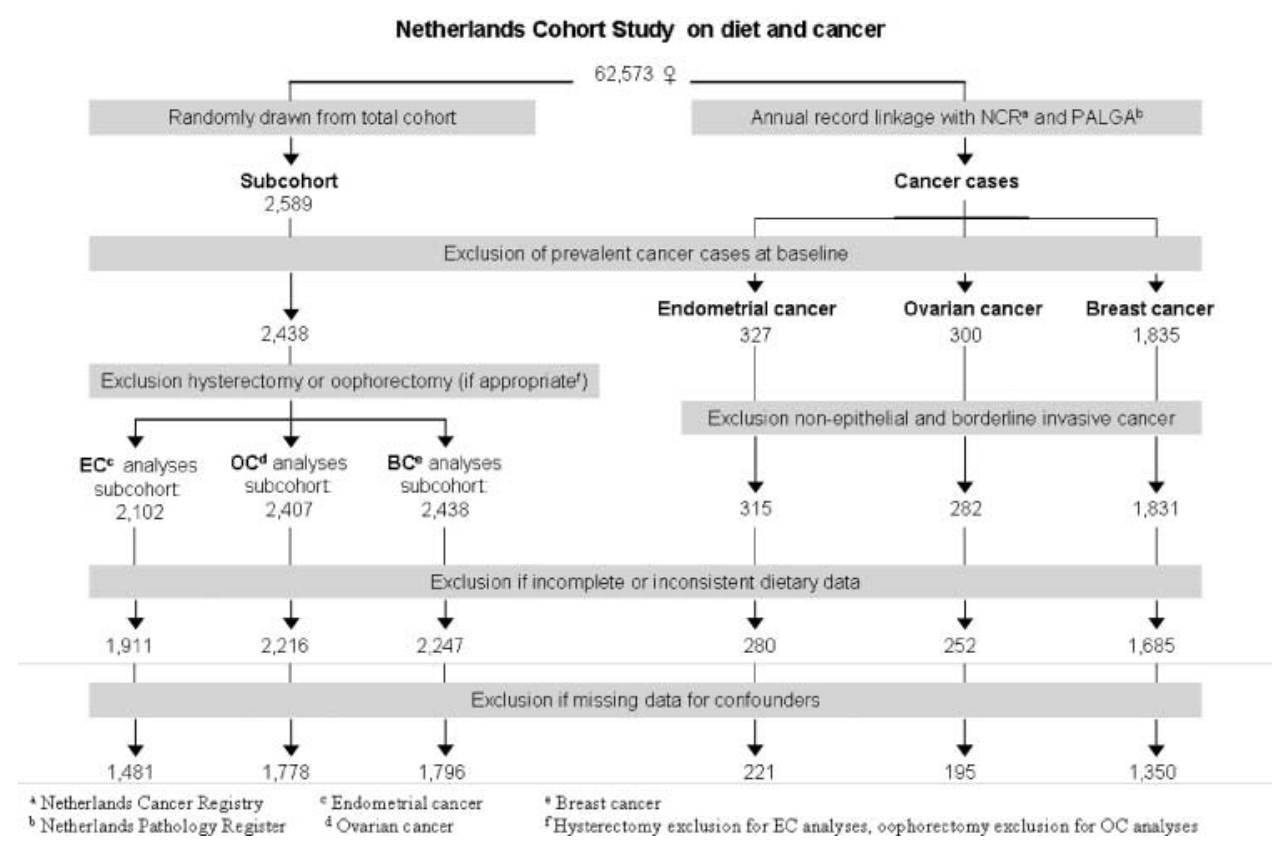

prospective cohort studies. In the present study, we investigated the relationship between dietary acrylamide and cancer of the endometrium, ovaries, and breasts within the framework of the Netherlands Cohort Study on diet and cancer (NLCS). These cancer types were chosen because of their association with acrylamide in animal studies and because there are indications of a positive association between fried food consumption and both ovarian and breast cancer $(24,25)$. We did subgroup analyses for women who reported to have never smoked to eliminate the influence of smoking, which is a major source of acrylamide exposure.

\section{Materials and Methods}

Study Cohort, Cases, and Follow-up. The Netherlands Cohort Study on diet and cancer started in September 1986 with the enrollment of 62,573 women aged 55 to 69 years. All of these women were presumed to be postmenopausal. At baseline, data on dietary habits and other risk factors were collected by means of a selfadministered questionnaire. The informed consent for this study consisted of the completing and returning of the questionnaire in 1986. Participants were informed that by returning a completed questionnaire, they gave consent to participate in a study about the etiology of cancer and for record linkage to the Netherlands Cancer Registry and the Netherlands Pathology Registry. This procedure of informed consent was approved by the Medical Ethics Committees of the University Hospital Maastricht and TNO Nutrition in February 1985 and July 1986, respectively.

Data processing and analysis were based on the casecohort approach because the desired level of detail of the questionnaire (e.g., including open-ended questions) prohibited high-throughput processing. Therefore, the questionnaires were not processed for the total cohort, but only for a subcohort and the cases. Following the case-cohort approach, cases were enumerated for the entire cohort (providing the numerator information for estimating incidence rates), whereas the accumulated person-years for the entire cohort were estimated from a subcohort of 2,589 women randomly sampled from the entire cohort at baseline (providing the denominator information for estimating incidence rates). Thus, all the incident cases were used in the analyses. The size of the subcohort was based on calculations that were described in a previous publication (26). Since the start of the study, the subcohort has been followed up regularly for vital status information. Incident cases in the total cohort have been detected by annual computerized record linkages to the regional cancer registries and the Netherlands Pathology Registry. The completeness of cancer followup through linkage with the national cancer registry was estimated to be at least $96 \%$ (27). The follow-up of the subcohort was $100 \%$ complete at the end of the follow-up period. Further details on the design of the study and methods of follow-up are presented elsewhere (26, 28-30).

The analyses are based on 11.3 years of follow-up, from September 1986 to December 1997. This is the most recent data set available for analysis because cancer registry procedures, linkage to the cancer registry, checks of the reported linkages, and the processing of the foodfrequency questionnaire cause considerable delays. After 11.3 years of follow-up, there were 327, 300, and 1,835 incident, microscopically confirmed, primary carcinomas of the endometrium ([ICD-O]-3:C54), ovaries ([ICD-O]3:C56.9), and breasts ([ICD-O]-3:C50), respectively. Cases were excluded from analysis if they had been diagnosed with non-epithelial tumors and borderline invasive tumors, and if their dietary data were incomplete or inconsistent.Subcohortmemberswereexcluded from 
Table 1. Acrylamide levels in foods, used for the NLCS acrylamide intake assessment

\begin{tabular}{|c|c|c|c|c|}
\hline & $n$ samples & Mean $(\mu \mathrm{g} / \mathrm{kg})$ & Minimum $(\mu \mathrm{g} / \mathrm{kg})$ & Maximum $(\mu \mathrm{g} / \mathrm{kg})$ \\
\hline Potato crisps & 40 & 1,249 & 310 & 2,800 \\
\hline Dutch spiced cake & 13 & 1,018 & 260 & 1,410 \\
\hline French fries & 33 & 351 & $<\mathrm{LOQ}^{*}$ & 1,220 \\
\hline Salty snacks & 12 & 277 & 45 & 867 \\
\hline Crisp bread & 12 & 229 & 15 & 914 \\
\hline Cookies & 20 & 204 & 10 & 829 \\
\hline Corn flakes & 12 & 121 & $<\mathrm{LOQ}^{*}$ & 300 \\
\hline Peanut butter & 2 & 113 & 107 & 118 \\
\hline Chocolate & 6 & 60 & 22 & 116 \\
\hline Nuts & 8 & 33 & $<\mathrm{LOQ}^{\dagger}$ & 83 \\
\hline Rusk & 3 & 25 & 16 & 33 \\
\hline Rye bread & 13 & 24 & $<\mathrm{LOQ}_{+}^{\ddagger}$ & 60 \\
\hline Sweet pastry & 19 & 18 & $<\operatorname{LOQ}^{\dagger}$ & 111 \\
\hline Coffee & 9 & 17 & 9 & 28 \\
\hline Chocolate milk & 4 & $<\mathrm{LOQ}^{\dagger}$ & $<\mathrm{LOQ}^{\dagger}$ & $<\mathrm{LOQ}^{\dagger}$ \\
\hline Bread & 22 & $<\operatorname{LOQ}^{\dagger}$ & $<\operatorname{LOQ}^{\dagger}$ & $<\operatorname{LOQ}^{\dagger}$ \\
\hline
\end{tabular}

*Limit of quantitation in 2002: $60 \mu \mathrm{g} / \mathrm{kg}$.

†Limit of quantitation in 2005: $15 \mu \mathrm{g} / \mathrm{kg}$.

ŁLimit of quantitation in 2002: $30 \mu \mathrm{g} / \mathrm{kg}$.

the analysis if they had been diagnosed with cancer other than skin cancer at baseline, if information on dietary habits was missing or inconsistent, and if they reported at baseline to have had a hysterectomy (excluded from the endometrial cancer analysis) or an oophorectomy (excluded from the ovarian cancer analysis). Figure 1 shows the selection and exclusion steps that resulted in the numbers of cases and subcohort members that were available for analysis.
Acrylamide Intake Assessment. In the NLCS, a food frequency questionnaire with questions on 150 food items was used to estimate dietary habits (29). The acrylamide intake was estimated from the mean acrylamide level of food items, and the frequency of consumption and portion size of the food items.

For the acrylamide intake estimation in the NLCS, we focused on foods that are likely to contain considerable acrylamide concentrations according to the mechanism
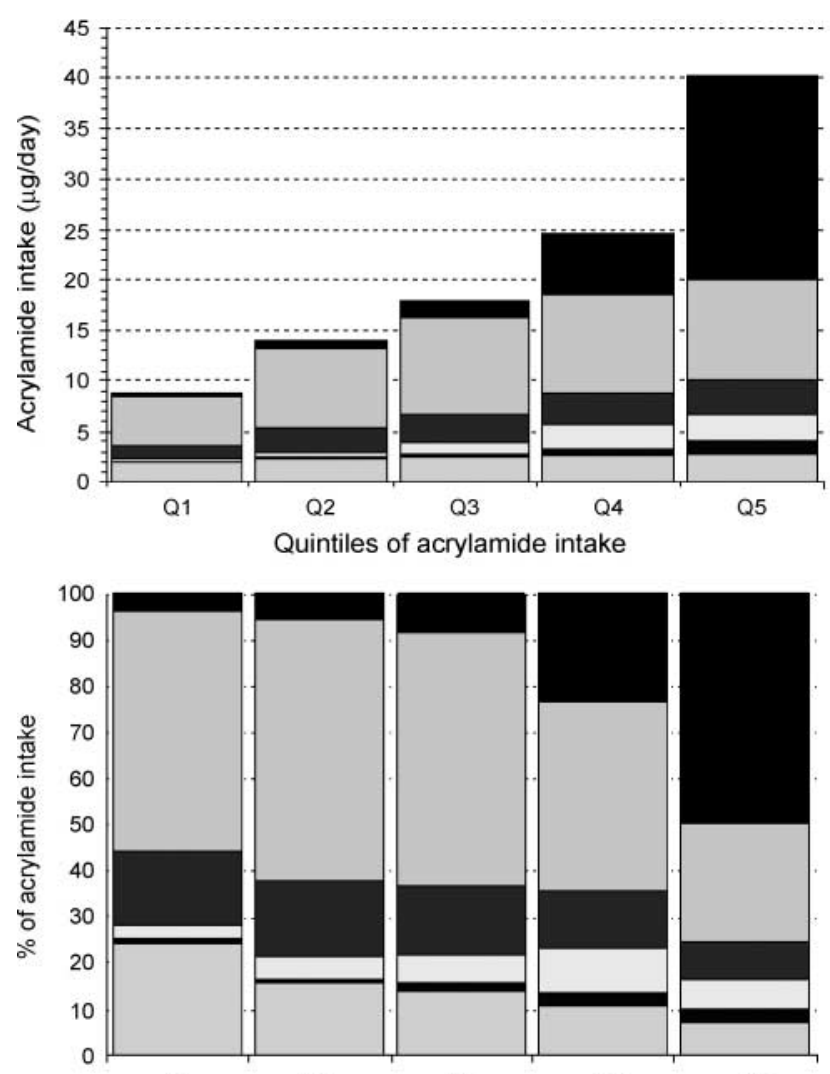

Q1

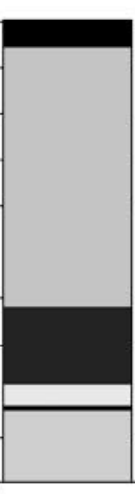

Q2

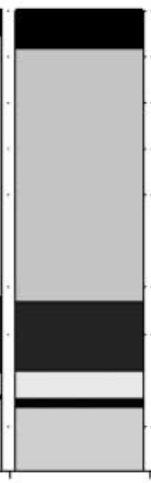

Q3

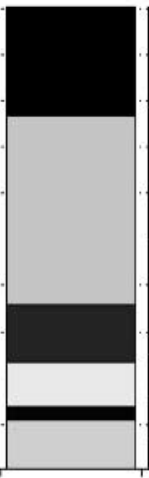

Q4

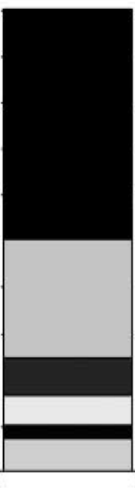

Q5
- Spiced honey cake

$\square$ Coffee

- Cookies

$\square$ French fries

- Crisps

$\square$ Other foods

Quintiles of acrylamide intake

Figure 2. Absolute and relative contribution of foods to the mean daily dietary acrylamide intake of the NLCS subcohort. 
Table 2. Characteristics of the subcohort members across quintiles of daily dietary acrylamide intake; the Netherlands Cohort Study on diet and cancer (NLCS), 1986 to 1997

\begin{tabular}{|c|c|c|c|c|c|c|}
\hline Variable & Q1 & Q2 & Q3 & Q4 & Q5 & $P^{*}$ \\
\hline Number of subcohort members & 471 & 482 & 455 & 466 & 470 & \\
\hline \multicolumn{7}{|l|}{ Dietary variables } \\
\hline Acrylamide intake, $\mu \mathrm{g} / \mathrm{d}^{\dagger}$ & $9.5(3.7)$ & $14.0(1.5)$ & $17.9(2.2)$ & $24.3(4.7)$ & $36.8(9.6)$ & $<0.001$ \\
\hline Acrylamide intake, $\mu \mathrm{g} / \mathrm{kg}_{+}$body weight per day ${ }^{\dagger}$ & $0.14(0.06)$ & $0.20(0.04)$ & $0.26(0.05)$ & $0.34(0.08)$ & $0.53(0.19)$ & $<0.001$ \\
\hline Coffee consumption, $\mathrm{g} / \mathrm{d}^{\dagger}$ & $250(125)$ & $500(125)$ & $500(250)$ & $500(375)$ & $500(375)$ & $<0.001$ \\
\hline Dutch spiced cake consumption, $\mathrm{g} / \mathrm{d}^{\dagger}$ & $0.0(0.0)$ & $0.0(0.7)$ & $0.0(1.8)$ & $1.8(7.8)$ & $20.4(12.6)$ & $<0.001$ \\
\hline Cookie consumption, $\mathrm{g} / \mathrm{d}$ & $3.7(8.7)$ & $9.6(20.4)$ & $16.7(14.8)$ & $16.7(14.8)$ & $16.7(14.8)$ & $<0.001$ \\
\hline Potato crisp consumption, $\mathrm{g} / \mathrm{d}^{\dagger}$ & $0.0(0.0)$ & $0.0(0.0)$ & $0.0(0.0)$ & $0.0(0.3)$ & $0.0(0.7)$ & $<0.001$ \\
\hline French fries consumption, $\mathrm{g} / \mathrm{d}^{\dagger}$ & $0.0(0.0)$ & $0.0(0.0)$ & $0.0(4.8)$ & $0.0(12.3)$ & $0.0(9.7)$ & $<0.001$ \\
\hline Total energy intake, $\mathrm{kcal}$ & $1467(449)$ & $1580(452)$ & $1671(465)$ & $1730(550)$ & $1821(548)$ & $<0.001$ \\
\hline Carbohydrate intake, $\mathrm{g} / \mathrm{d}_{+}^{\dagger}$ & $153(55)$ & $162(50)$ & $176(53)$ & $183(61)$ & $200(69)$ & $<0.001$ \\
\hline Saturated fat intake, $\mathrm{g} / \mathrm{d}^{\dagger}$ & $25.5(12.0)$ & $28.0(12.6)$ & $28.9(11.6)$ & $29.8(13.2)$ & $30.8(12.3)$ & $<0.001$ \\
\hline Trans-unsaturated fatty acid intake, $\mathrm{g} / \mathrm{d}^{\dagger}$ & $1.8(1.2)$ & $2.1(1.3)$ & $2.3(1.4)$ & $2.6(1.5)$ & $2.7(1.6)$ & $<0.001$ \\
\hline Fiber intake, $\mathrm{g} / \mathrm{d}^{\dagger}$ & $22.9(9.5)$ & $23.9(8.5)$ & $24.5(9.1)$ & $25.7(9.6)$ & $26.6(8.6)$ & $<0.001$ \\
\hline Vegetable intake, $\mathrm{g} / \mathrm{d}^{\dagger}$ & $184(107)$ & $182(102)$ & $179(83)$ & $190(104)$ & $184(96)$ & 0.34 \\
\hline Fruit intake, $\mathrm{g} / \mathrm{d}$ & $184(151)$ & 179 (149) & $175(140)$ & $174(138)$ & $180(146)$ & 0.42 \\
\hline Alcohol intake, $\mathrm{g} / \mathrm{d}^{\dagger}$ & $0.9(8.4)$ & $1.6(7.8)$ & $2.0(8.6)$ & $1.9(7.0)$ & $1.4(7.1)$ & 0.09 \\
\hline \multicolumn{7}{|l|}{ Nondietary variables } \\
\hline Age, $y^{\dagger}$ & $62(7)$ & $61(7)$ & $60(7)$ & $61(8)$ & $61(7)$ & $<0.001$ \\
\hline BMI, $\mathrm{kg} / \mathrm{m}_{+}^{2+}$ & $24.6(4.3)$ & $24.8(4.4)$ & $24.8(4.3)$ & $24.6(4.3)$ & $24.3(4.2)$ & 0.07 \\
\hline Height, $\mathrm{cm}^{\dagger}$ & $165(8)$ & $165(9)$ & $165(8)$ & $165(8)$ & $165(10)$ & 0.18 \\
\hline Parity, $n$ children $^{\dagger}$ & $2(3)$ & $3(3)$ & $3(2)$ & $3(2)$ & $2(3)$ & 0.003 \\
\hline Age at menarche, $\mathrm{y}^{\dagger}$ & $13(2)$ & $13(2)$ & $14(3)$ & $14(2)$ & $14(3)$ & 0.12 \\
\hline Age at menopause, $\mathrm{y}^{\dagger}$ & $50(6)$ & $50(6)$ & $49(7)$ & $50(6)$ & $50(6)$ & 0.38 \\
\hline Age at first childbirth, $\mathrm{y}^{\dagger}$ & $27(5)$ & $26(5)$ & $27(5)$ & $26(5)$ & $26(5)$ & 0.92 \\
\hline Current cigarette smoking, $\%$ yes ${ }^{\ddagger}$ & 17.0 & 18.9 & 25.9 & 21.5 & 19.2 & 0.02 \\
\hline$n$ cigarettes per day among ever-smokers ${ }^{\dagger}$ & $10(15)$ & $10(15)$ & $10(15)$ & $10(10)$ & $10(15)$ & 0.79 \\
\hline$n$ smoking years among ever-smokers ${ }^{\dagger}$ & $31(20)$ & $27(20)$ & $30(20)$ & $28(21)$ & 29 (19) & 0.03 \\
\hline Non-occupational physical activity, minutes $/ \mathrm{d}^{\dagger}$ & $51.4(57.9)$ & $51.4(54.6)$ & $53.6(54.3)$ & $53.6(53.6)$ & $51.4(60.0)$ & 0.91 \\
\hline Oral contraceptive use, $\%$ ever & 22.6 & 25.1 & 26.1 & 26.5 & 23.6 & 0.61 \\
\hline Oral contraceptive use duration among ever-users, $\mathrm{y}^{\dagger}$ & $6(9)$ & $6(9)$ & $6(8)$ & $7(10)$ & $8(8)$ & 0.22 \\
\hline Postmenop. hrt $^{\S}$ use, \% ever ${ }^{\ddagger}$ & 13.6 & 14.9 & 12.6 & 11.4 & 11.0 & 0.76 \\
\hline Postmenop. $\mathrm{hrt}^{8}$ use duration among ever-users, $\mathrm{y}^{\dagger}$ & $2(5)$ & $1(4)$ & $1(2)$ & $2(3)$ & $2(4)$ & 0.15 \\
\hline Education, $\%^{\ddagger}$ & & & & & & $<0.001$ \\
\hline Primary school & 29.9 & 30.9 & 34.5 & 35.2 & 35.1 & \\
\hline Lower vocational school & 18.3 & 20.5 & 25.7 & 26.2 & 24.0 & \\
\hline Intermediate vocational/high school & 37.2 & 39.6 & 33.6 & 29.0 & 32.3 & \\
\hline Higher vocational school/University & 13.6 & 8.5 & 5.5 & 9.0 & 8.1 & \\
\hline Family history of breast cancer, $\%$ yes ${ }^{\ddagger}$ & 8.5 & 8.9 & 7.9 & 7.5 & 11.1 & 0.35 \\
\hline Family history of endometrial cancer, $\%$ yes ${ }^{\ddagger}$ & 2.3 & 2.3 & 3.5 & 3.4 & 2.3 & 0.60 \\
\hline Family history of ovarian cancer, $\%$ yes & 0.0 & 0.0 & 0.0 & 0.2 & 0.0 & 0.52 \\
\hline
\end{tabular}

${ }^{*} P$ value for Kruskal-Wallis test or $\chi^{2}$ test (proportions).

tData represent medians (interquartile range).

$\ddagger$ Percentages.

$\S$ Postmenop.hrt $=$ postmenopausal hormone treatment.

of formation and reports from various European countries (31). Thus, the following list of food products was established: French fries, potato crisps, bread and rye bread, cookies, pastry, rusk, crisp bread, chocolate and chocolate milk, nuts, peanut butter, breakfast cereals, and coffee. To obtain an intake estimate representative for the Dutch situation, we used data on acrylamide levels in food products on the Dutch market. In 2002, the Dutch Food and Consumer Product Safety Authority analyzed acrylamide levels in various foods, such as bread, French fries, pastry and cake, Dutch spiced cake, crisps, corn flakes, meat, fish, and several beverages, following an elaborate sampling scheme (5). In 2005, another series of foods was analyzed to specifically accommodate the estimation of acrylamide intake of the NLCS cohort, and this series consisted of bread, rusk, specific types of biscuits, rye bread, crisp bread, pastry and cake, chocolate and chocolate milk, nuts and salty snacks, peanut butter, and coffee (31). Five types of bread were analyzed again in 2005 because the quantitation limit of the analytic method had decreased from $30 \mathrm{ppb}$ (even 60 ppb for some foods) in 2002 to 15 ppb in 2005, which offered the opportunity to more accurately estimate the acrylamide content of bread. Acrylamide in coffee was analyzed in brewed filtered coffee that was prepared according to general Dutch preparation instructions ( $7 \mathrm{~g}$ of coffee powder $/ 125 \mathrm{~mL}$ of water).

Acrylamide measurements in cookies were done in several types of cookies known to be eaten most frequently by the population comparable to the NLCS, according to information from the development phase of the questionnaire. Thus, an acrylamide level for cookies was based on the acrylamide level of the specific types of cookies weighted by the frequency of consumption of the NLCS-comparable population. The same was done for other composite food items, such as pies and chocolates.

The foods listed above were assigned the mean value of the acrylamide values per food or a value half the quantitation limit when levels were lower than the 
quantitation limit because they probably do contain some acrylamide. Other foods (meats, cooked and raw vegetables, dairy products) were assigned the value zero. Table 1 shows the acrylamide levels of foods that were used in the acrylamide intake assessment.

Statistical Analysis. Acrylamide was included in the statistical models as a continuous variable and as quintiles.
Covariables were included in the models if they changed age-adjusted regression coefficients of acrylamide by more than $10 \%$. A priori, the following variables were considered as potential confounders: age at menarche, duration of oral contraceptive use, age at first childbirth, parity, age at menopause, duration of postmenopausal hormone use, non-occupational physical activity, body mass index (BMI), height,

Table 3. Age and multivariable-adjusted association between acrylamide intake and risk of endometrial, ovarian and breast cancer; the Netherlands Cohort Study on diet and cancer (NLCS), 1986 to 1997

\begin{tabular}{|c|c|c|c|c|c|c|c|}
\hline & $\begin{array}{l}\text { Increment per } 10 \mu \mathrm{g} \\
\text { acrylamide/d }\end{array}$ & Q1 & Q2 & Q3 & Q4 & Q5 & $P_{\text {trend }}$ \\
\hline \multicolumn{8}{|l|}{$\begin{array}{l}\text { Endometrial cancer } \\
\text { All }\end{array}$} \\
\hline $\begin{array}{l}\text { Cases / person-years* } \\
\text { HR }(95 \% \text { CI }) \\
\text { HR }(95 \% \text { CI })^{\S} \\
\text { Endometrial cancer }\end{array}$ & $\begin{array}{c}221 / 15,836 \\
1.01(0.90-1.14) \\
1.04(0.91-1.19)\end{array}$ & $\begin{array}{c}44 / 3,202 \\
1.00^{\ddagger} \\
1.00^{\ddagger}\end{array}$ & $\begin{array}{c}40 / 3,078 \\
0.96(0.61-1.51) \\
0.95(0.59-1.54)\end{array}$ & $\begin{array}{c}39 / 3,095 \\
0.94(0.59-1.49) \\
0.94(0.56-1.56)\end{array}$ & $\begin{array}{c}46 / 3,175 \\
1.09(0.70-1.70) \\
1.21(0.74-1.98)\end{array}$ & $\begin{array}{c}52 / 3,285 \\
1.17(0.76-1.79) \\
1.29(0.81-2.07)\end{array}$ & $\begin{array}{l}0.38 \\
0.18\end{array}$ \\
\hline \multicolumn{8}{|l|}{ Never-smokers } \\
\hline $\begin{array}{l}\text { Cases/person-years*, } \\
\text { never-smokers } \\
\text { HR }(95 \% \text { CI) } \\
\text { HR }(95 \% \text { CI })^{\S} \\
\text { Ovarian cancer } \\
\text { All }\end{array}$ & $\begin{array}{c}150 / 9,422 \\
1.07(0.93-1.24) \\
1.12(0.95-1.33)\end{array}$ & $\begin{array}{l}27 / 2,113 \\
1.00^{\ddagger} \\
1.00^{\ddagger}\end{array}$ & $\begin{array}{l}1.10(0.62-1.97) \\
1.16(0.63-2.15)\end{array}$ & $\begin{array}{l}1.36(0.77-2.40) \\
1.35(0.73-2.51)\end{array}$ & $\begin{array}{l}1.23(0.70-2.16) \\
1.30(0.69-2.46)\end{array}$ & $\begin{array}{c}40 / 1,931 \\
1.63(0.97-2.74) \\
1.99(1.12-3.52)\end{array}$ & $\begin{array}{l}0.07 \\
0.03\end{array}$ \\
\hline $\begin{array}{l}\text { Cases/person-years* } \\
\text { HR }(95 \% \text { CI) } \\
\text { HR }(95 \% \text { CI })^{\|} \\
\text {Ovarian cancer }\end{array}$ & $\begin{array}{c}195 / 19,037 \\
1.11(0.99-1.24) \\
1.11(0.99-1.25)\end{array}$ & $\begin{array}{c}31 / 3,803 \\
1.00^{\ddagger} \\
1.00^{\ddagger}\end{array}$ & $\begin{array}{c}39 / 3,939 \\
1.26(0.77-2.06) \\
1.22(0.73-2.01)\end{array}$ & $\begin{array}{c}32 / 3,595 \\
1.16(0.69-1.94) \\
1.12(0.65-1.92)\end{array}$ & $\begin{array}{c}39 / 3,823 \\
1.33(0.82-2.18) \\
1.28(0.77-2.13)\end{array}$ & $\begin{array}{c}54 / 3,877 \\
1.77(1.11-2.82) \\
1.78(1.10-2.88)\end{array}$ & $\begin{array}{l}0.02 \\
0.02\end{array}$ \\
\hline \multicolumn{8}{|l|}{ Never-smokers } \\
\hline $\begin{array}{l}\text { Cases/person-years*, } \\
\text { never-smokers } \\
\text { HR( } 95 \% \text { CI) } \\
\text { HR }\left(95 \% \text { CI }{ }^{\|}\right. \\
\text {Breast cancer } \\
\text { All }\end{array}$ & $\begin{array}{c}129 / 11,446 \\
1.16(1.00-1.34) \\
1.17(1.01-1.36)\end{array}$ & $\begin{array}{l}18 / 2,540 \\
1.00^{\ddagger} \\
1.00^{\ddagger}\end{array}$ & $\begin{array}{c}27 / 2,373 \\
1.62(0.87-3.02) \\
1.60(0.85-3.02)\end{array}$ & $\begin{array}{l}1.64(0.85-3.16) \\
1.64(0.84-3.19)\end{array}$ & $\begin{array}{c}29 / 2,250 \\
1.90(1.03-3.50) \\
1.86(1.00-3.48)\end{array}$ & $\begin{array}{c}33 / 2,292 \\
\\
2.06(1.13-3.77) \\
2.22(1.20-4.08)\end{array}$ & $\begin{array}{l}0.02 \\
0.01\end{array}$ \\
\hline $\begin{array}{l}\text { Cases / person-years* } \\
\text { HR }(95 \% \text { CI })^{\dagger} \\
\text { HR }(95 \% \text { CI })^{\pi} \\
\text { Breast cancer }\end{array}$ & $\begin{array}{c}1,350 / 19,036 \\
0.98(0.92-1.04) \\
0.99(0.92-1.06)\end{array}$ & $\begin{array}{c}296 / 3,732 \\
1.00^{\ddagger} \\
1.00^{\ddagger}\end{array}$ & $\begin{array}{c}267 / 3,941 \\
0.86(0.69-1.07) \\
0.80(0.64-1.02)\end{array}$ & $\begin{array}{c}257 / 3,616 \\
0.91(0.72-1.14) \\
0.92(0.72-1.17)\end{array}$ & $\begin{array}{c}254 / 3,877 \\
0.84(0.67-1.05) \\
0.86(0.67-1.10)\end{array}$ & $\begin{array}{c}276 / 3,871 \\
0.90(0.73-1.13) \\
0.93(0.73-1.19)\end{array}$ & $\begin{array}{l}0.37 \\
0.79\end{array}$ \\
\hline \multicolumn{8}{|l|}{ Never-smokers } \\
\hline $\begin{array}{l}\text { Cases/person-years*, } \\
\text { never-smokers } \\
\text { HR(95\% CI })^{\dagger} \\
\text { HR }(95 \% \text { CI })^{\uparrow}\end{array}$ & $\begin{array}{c}767 / 11,540 \\
1.01(0.93-1.09) \\
1.01(0.93-1.11)\end{array}$ & $\begin{array}{c}159 / 2,496 \\
1.00^{\ddagger} \\
1.00^{\ddagger}\end{array}$ & $\begin{array}{c}159 / 2,379 \\
1.06(0.80-1.41) \\
0.97(0.72-1.32)\end{array}$ & $\begin{array}{c}147 / 2,054 \\
1.18(0.88-1.59) \\
1.17(0.85-1.61)\end{array}$ & $\begin{array}{c}144 / 2,317 \\
1.01(0.75-1.36) \\
1.00(0.73-1.38)\end{array}$ & $\begin{array}{c}158 / 2,294 \\
1.10(0.82-1.47) \\
1.10(0.80-1.52)\end{array}$ & $\begin{array}{l}0.66 \\
0.55\end{array}$ \\
\hline
\end{tabular}

NOTE: The numbers of cases and person-years are the numbers that resulted after list-wise deletion of observations with missing values on the selected confounders.

${ }^{*}$ Number of cases that accumulated in the total cohort/person-years at risk of the subcohort.

†Age-adjusted (same data set as in multivariable-adjusted analyses).

‡Reference category.

§Adjusted for: age (y), age at menarche (y), age at menopause (y), age at first childbirth (dummy: nulliparous, 17-19 y, 20-24 y, 25-29 y, $\geq 30 \mathrm{y})$, parity $(n$ children), duration of oral contraceptives use (y), duration of postmenopausal hormone use (y), BMI $\left(\mathrm{kg} / \mathrm{m}^{2}\right)$, height $(\mathrm{cm})$, current smoking (yes/no), quantity of smoking (cigarettes/d), duration of smoking ( $n$ smoking years), non-occupational physical activity (min/d), energy intake (kcal/d), transunsaturated fatty acid intake (energy-adjusted; g/d), carbohydrate intake (energy-adjusted; g/d), alcohol consumption (g/d).

$\|$ Adjusted for age (y), age at menarche (y), age at menopause (y), parity ( $n$ children), duration of oral contraceptives use (y), duration of postmenopausal hormone use $(\mathrm{y})$, BMI $\left(\mathrm{kg} / \mathrm{m}^{2}\right)$, height $(\mathrm{cm})$, current smoking (yes/no), quantity of smoking (cigarettes/d), duration of smoking ( $n$ smoking years), saturated fat intake (energy-adjusted; g/d), trans-unsaturated fatty acid intake (energy-adjusted; g/d).

TAdjusted for age (y), age at menarche (y), age at menopause (y), age at first childbirth (dummy: nulliparous, 17-19 y, 20-24 y, 25-29 y, $\geq 30 \mathrm{y}$ ), duration of oral contraceptives use (y), duration of postmenopausal hormone use $(\mathrm{y}), \mathrm{BMI}\left(\mathrm{kg} / \mathrm{m}^{2}\right)$, height $(\mathrm{cm})$, current smoking (yes/no), quantity of smoking (cigarettes/d), duration of smoking ( $n$ smoking years), socioeconomic status (four levels of education), energy intake (kcal/d), saturated fat intake (energyadjusted; g/d), carbohydrate intake (energy-adjusted; g/d), family history of breast cancer (yes/no), history of benign breast disease (yes/no). 
education level, family history of endometrial cancer (in the endometrial cancer analyses), ovarian or breast cancer (in the ovarian cancer analyses), breast cancer (in the breast cancer analyses), and energy intake (32). We also checked the following broad categories of foods or nutrients for confounding potential: alcohol con- sumption, intake of vegetables and fruits, and energyadjusted intake of saturated fat, trans-unsaturated fatty acids, carbohydrates, and dietary fiber. Smoking status (current versus not current), the duration of smoking, and the number of cigarettes per day were always included in the model because cigarette smoke is a very

Table 4. Association between acrylamide intake and risk of endometrial and ovarian cancer, adjusted for acrylamide-containing foods; the Netherlands Cohort Study on diet and cancer (NLCS), 1986 to 1997

$\begin{array}{llllllll}\begin{array}{c}\text { Additionally } \\ \text { adjusted for }\end{array} & \begin{array}{l}\text { Increment } \\ \text { per } 10 \mu \mathrm{g} \\ \text { acrylamide/d }\end{array} & \text { Q1 } & \text { Q2 } & \text { Q3 } & \text { Q4 } & \text { Q5 } & P_{\text {trend }} \\ \end{array}$

Endometrial cancer

All

\begin{tabular}{|c|c|c|c|c|c|c|c|c|}
\hline Cases/person-years* & & $221 / 15,836$ & $44 / 3,202$ & $40 / 3,078$ & $39 / 3,095$ & $46 / 3,175$ & $52 / 3,285$ & \\
\hline HR $(95 \% \text { CI })_{+}^{\dagger}$ & na & $1.04(0.91-1.19)$ & $1.00^{\ddagger}$ & $0.95(0.59-1.54)$ & $0.94(0.56-1.56)$ & $1.21(0.74-1.98)$ & $1.29(0.81-2.07)$ & 0.18 \\
\hline $\mathrm{HR}(95 \% \mathrm{CI})^{\dagger}$ & Coffee & $1.06(0.93-1.22)$ & $1.00^{\ddagger}$ & $1.02(0.61-1.71)$ & $1.04(0.60-1.87)$ & $1.35(0.79-2.32)$ & $1.46(0.86-2.46)$ & 0.09 \\
\hline $5 \% \mathrm{CI})^{\dagger}$ & Dutch spiced cake & 1.07 & $1.00^{\ddagger}$ & 0.96 & $0.96(C$ & 1.36 & $6-3.97)$ & 0.17 \\
\hline$\% \mathrm{CI})^{\dagger}$ & Cookies & $1.05(0.92-1.20)$ & $1.00^{\S}$ & 0.98 ( & $0.98(0.58-1.65)$ & $1.27(0.77-2.09)$ & $1.37(0.84-2.21)$ & 0.13 \\
\hline$\% \% \mathrm{CI})_{+}^{\dagger}$ & French fries & $1.01(0.88-1.17)$ & $1.00_{+}^{\ddagger}$ & $0.95(0.59-1.54)$ & $0.93(0.56-1.55)$ & $1.16(0.71-1.92)$ & $1.24(0.76-2.00)$ & 0.29 \\
\hline$\% \mathrm{CI})^{\dagger}$ & Potato crisps & $1.03(0.90-1.19)$ & $1.00^{\ddagger}$ & $0.95(0.59-1.54)$ & $0.94(0.56-1.56)$ & $1.20(0.73-1.97)$ & $1.28(0.80-2.06)$ & 0.20 \\
\hline
\end{tabular}

Never-smokers

\begin{tabular}{|c|c|c|c|c|c|c|c|c|}
\hline $\begin{array}{c}\text { Cases/person-years*, } \\
\text { never-smokers }\end{array}$ & & $150 / 9,422$ & $27 / 2,113$ & $25 / 1,786$ & $29 / 1,709$ & $29 / 1,883$ & $40 / 1,931$ & \\
\hline $\operatorname{HR}(95 \% \mathrm{CI})^{\dagger}$ & na & $1.12(0.95-1.33)$ & $1.00_{+}^{\ddagger}$ & $1.16(0.63-2.15)$ & $1.35(0.73-2.51)$ & $1.30(0.69-2.46)$ & 1.99 (1.12-3.52) & 0.03 \\
\hline $\mathrm{HR}(95 \% \mathrm{CI})^{\dagger}$ & Coffee & $1.13(0.95-1.35)$ & $1.00^{\ddagger}$ & 1.25( & & $1.44(0.14-2.19)$ & $2.16(1.16-4.05)$ & 0.02 \\
\hline $\mathrm{HR}(95 \% \mathrm{CI})^{\dagger}$ & Dutch spiced cake & $1.05(0.71-1.55)$ & $1.00^{\ddagger}$ & $1.17(0.64-2.17)$ & $1.38(0.75-2.57)$ & $1.45(0.73-2.88)$ & $2.67(1.08-6.61)$ & 0.08 \\
\hline $\mathrm{HR}(95 \% \mathrm{CI})_{+}^{\dagger}$ & Cookies & $1.14(0.96-1.35)$ & $1.00_{+}^{\ddagger}$ & $1.23(0.66-2.27)$ & $1.45(0.77-2.75)$ & $1.39(0.74-2.63)$ & $2.13(1.18-3.85)$ & 0.02 \\
\hline $\mathrm{HR}(95 \% \mathrm{CI})^{\dagger}$ & French fries & $1.11(0.93-1.32)$ & $1.00^{\ddagger}$ & 1.16 & & $1.26(0.6$ & $1.92(1.07-3.45)$ & 0.05 \\
\hline HR $(95 \% \mathrm{CI})^{\dagger}$ & Potato crisps & $1.14(0.95-1.35)$ & $1.00^{\ddagger}$ & $1.16(0.63-2.14)$ & $1.35(0.73-2.51)$ & $1.32(0.70-2.48)$ & $2.01(1.14-3.57)$ & 0.02 \\
\hline
\end{tabular}

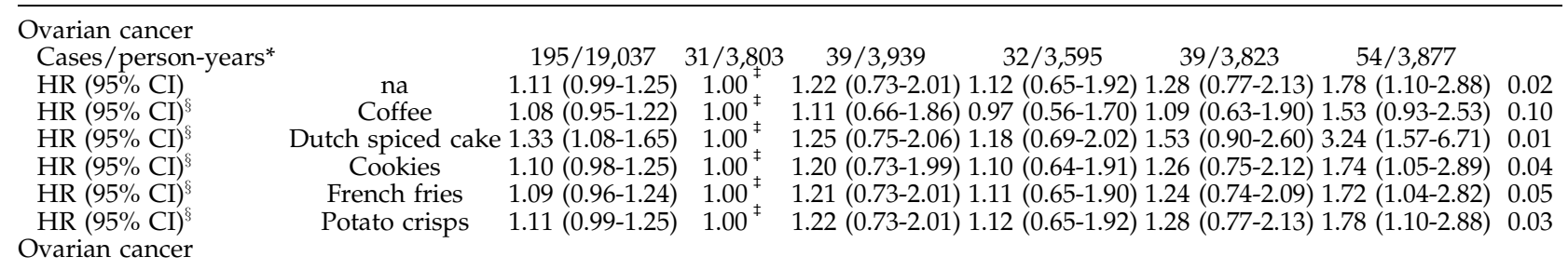

Never-smokers

\begin{tabular}{|c|c|c|c|c|c|c|c|c|}
\hline $\begin{array}{l}\text { Cases/person-years*, } \\
\text { never-smokers }\end{array}$ & & $129 / 11,446$ & $18 / 2,540$ & $27 / 2,373$ & $22 / 1,992$ & $29 / 2,250$ & $33 / 2,292$ & \\
\hline $\mathrm{HR}(95 \% \mathrm{CI})^{\mathrm{s}}$ & na & $1.17(1.01-1.36)$ & $1.00^{\ddagger}$ & $1.60(0.85-3.02)$ & $1.64(0.84-3.19)$ & $1.86(1.00-3.48)$ & $2.22(1.20-4.08)$ & 0.01 \\
\hline $\operatorname{HR}(95 \% \mathrm{CI})^{\S}$ & Coffee & $1.12(0.95-1.32)$ & $1.00_{+}^{\mp}$ & $1.43(0.75-2.75)$ & $1.37(0.68-2.76)$ & $1.54(0.77-3.05)$ & $1.86(0.98-3.51)$ & 0.08 \\
\hline HR $(95 \% \mathrm{CI})^{\S}$ & Dutch spiced cake & $1.44(1.07-1.94)$ & $1.00^{\ddagger}$ & $1.62(0.86-3.06)$ & $1.67(0.85-3.26)$ & 2.01 (1.04-3.88) & 2.91 (1.10-7.67) & 0.02 \\
\hline HR $(95 \% \text { CI })^{\S}$ & Cookies & $1.18(1.00-1.38)$ & $1.00_{+}^{\ddagger}$ & $1.63(0.87-3.06)$ & $1.68(0.85-3.32)$ & $1.92(1.01-3.65)$ & $2.29(1.21-4.34)$ & 0.02 \\
\hline $\operatorname{HR}(95 \% \mathrm{CI})^{\S}$ & French fries & 1.17 (1.01-1.37) & $1.00_{+}^{\mp}$ & $1.61(0.85-3.05)$ & $1.65(0.84-3.22)$ & $1.91(1.00-3.64)$ & $2.26(1.21-4.24)$ & 0.01 \\
\hline HR $(95 \% \mathrm{CI})^{\S}$ & Potato crisps & $1.17(1.00-1.36)$ & $1.00^{\ddagger}$ & $1.60(0.85-3.02)$ & $1.63(0.84-3.18)$ & $1.85(0.99-3.47)$ & 2.19 (1.19-4.04) & 0.01 \\
\hline
\end{tabular}

NOTE: Data represent HRs for acrylamide intake. The numbers of cases and person-years are the numbers that resulted after list-wise deletion of observations with missing values on the selected confounders. Number (percentage) of cases who consumed coffee: endometrial cancer cases: 212 (95.9); ovarian cancer cases: 192 (98.5). Number (percentage) of cases who consumed Dutch spiced cake: endometrial cancer cases: 110 (49.8); ovarian cancer cases: 104 (53.3). Number (percentage) of cases who consumed cookies: endometrial cancer cases: 194 (87.8); ovarian cancer cases: 179 (91.8). Number (percentage) of cases who consumed French fries: endometrial cancer cases: 82 (37.1); ovarian cancer cases: 56 (28.7). Number (percentage) of cases who consumed potato crisps: endometrial cancer cases: 51 (23.1); ovarian cancer cases: 42 (21.5).

Abbreviation: na, not applicable. Hrs are from Table 3.

${ }^{*}$ Number of cases that accumulated in the total cohort/person-years at risk of the subcohort.

$\dagger$ Adjusted for age (y), age at menarche (y), age at menopause (y), age at first childbirth (y), parity ( $n$ children), duration of oral contraceptives use (y), duration of postmenopausal hormone use $(\mathrm{y}), \mathrm{BMI}\left(\mathrm{kg} / \mathrm{m}^{2}\right)$, height $(\mathrm{cm})$, current smoking (yes/no), quantity of smoking (cigarettes/d), duration of smoking ( $n$ smoking years), non-occupational physical activity ( $\mathrm{min} / \mathrm{d})$, energy intake (kcal/d), trans-unsaturated fatty acid intake (energy-adjusted; g/d), carbohydrate intake (energy-adjusted; g/d), alcohol consumption (g/d).

$\ddagger$ Reference category.

§Adjusted for age (y), age at menarche (y), age at menopause (y), parity ( $n$ children), duration of oral contraceptives use (y), duration of postmenopausal hormone use $(\mathrm{y})$, BMI $\left(\mathrm{kg} / \mathrm{m}^{2}\right)$, height $(\mathrm{cm})$, current smoking (yes/no), quantity of smoking (cigarettes/d), duration of smoking ( $n$ smoking years), saturated fat intake (energy-adjusted; g/d), trans-unsaturated fatty acid intake (energy-adjusted; g/d). 
important source of acrylamide. Smokers have been shown to have on average four times higher levels of acrylamide-hemoglobin adducts, which is a marker of internal dose of acrylamide, than nonsmokers $(33,34)$. Also, for this reason, subgroup analyses were done for never-smokers.

The proportional hazards assumption was tested using scaled Schoenfeld residuals. Hazard rate ratios (HR) and confidence intervals were obtained through Cox proportional hazards regression with STATA software (package 9.2). SEs were estimated using the robust Huber-White sandwich estimator to account for additional variance introduced by sampling from the cohort. Tests for doseresponse trends were done by fitting ordinal exposure variables as continuous variables.

To investigate if the observed associations could be attributed to acrylamide specifically or whether the association was due to other characteristics of acrylamide-containing foods, models were run with acrylamide and the foods (one at a time) that explained most variance in acrylamide intake.

To check for the influence of protopathic bias, the analyses were also done with exclusion of cases occurring in the first 2 years of follow-up.

Effect modification of the association between acrylamide intake and cancer by other variables was tested using Wald $x^{2}$ tests. The variables that were tested for effect modification were selected based on their ability to modify the activity of CYP2E1, the enzyme that converts acrylamide to glycidamide. These variables are diabetes (ever/never diagnosis of diabetes), obesity $\left(\mathrm{BMI}>30 \mathrm{~kg} / \mathrm{m}^{2}\right)$, smoking (both duration of smoking in years and number of cigarettes smoked per day), alcohol consumption $(0,>0-5,>5 \mathrm{~g} /$ day $)$, and physical activity $(<30,30$ to $<60,60$ to $<90, \geq 90$ minutes/day; refs. 35-38).

Two-sided $P$ values are reported throughout this paper.

\section{Results}

On average, the subcohort members had a daily acrylamide intake of $21.0 \pm 11.9 \mu \mathrm{g}$, which corresponds to $0.32 \pm 0.19 \mu \mathrm{g}$ acrylamide per $\mathrm{kg}$ body weight per day. Although coffee was overall the most important contributor to acrylamide intake, Fig. 2 shows that not coffee, but Dutch spiced cake was chiefly responsible for the variation in acrylamide intake in this population, followed by coffee, French fries, potato crisps, and cookies.

Table 2 shows the values of the covariables that were assessed for confounding potential across acrylamide intake quintiles. From this table, it can be concluded that some variables differed considerably between the acrylamide intake categories. There were linear relationships between acrylamide intake and total energy, carbohydrate, saturated fat, trans-unsaturated fatty acids, and fiber intake. There were some significant differences in the nondietary variables between the quintiles of acrylamide intake, but these variables did not show a linear correlation with acrylamide intake.

In Table 3, the age-adjusted and multivariableadjusted associations between acrylamide intake and endometrial, ovarian, and breast cancer are shown. There was no statistically significant association between acrylamide as a continuous variable and endometrial cancer, and in the total group of women, there were also no statistically significant associations between quintiles of acrylamide intake and endometrial cancer. However, in the group of never-smoking women, the multivariable-adjusted $\mathrm{HR}$ in the fifth quintile [HR, 1.99; 95\% confidence interval (95\% CI), 1.12-3.52] was statistically significantly higher than that in the lowest quintile of acrylamide intake $\left(P_{\text {trend }}=0.03\right)$.

In the total group, acrylamide intake as a continuous variable was borderline significantly associated with ovarian cancer (HR, 1.11; 95\% CI, 0.99-1.25 for an increment of acrylamide intake with $10 \mu \mathrm{g} /$ day). In the never-smoker subgroup, the association was statistically significant (HR, 1.17; 95\% CI, 1.01-1.36 for an increment of acrylamide intake with $10 \mu \mathrm{g} /$ day). Also, the HR in the highest quintile of acrylamide intake was statistically significantly higher [total group: HR, 1.78 (1.10-2.88); never-smokers: HR, 2.22 (1.20-4.08)] than that of the lowest quintile, and the $P_{\text {trend }}$ was significant $(P=0.02$ and 0.01 , respectively).

There were no indications of an association between acrylamide intake and breast cancer.

Table 4 shows the multivariable-adjusted associations between acrylamide intake and endometrial and ovarian cancer additionally adjusted for coffee, Dutch spiced cake, cookies, French fries, and potato crisps consumption, respectively. For both endometrial and ovarian cancer, adjustment for Dutch spiced cake increased the HRs in the highest quintiles, and for ovarian cancer, it also increased the HR of the continuous acrylamide variable. Adjustment for coffee intake slightly decreased the HRs of the highest quintiles of acrylamide intake and the continuous acrylamide variable for ovarian cancer only. Adjustment for the other foods did not materially change the HRs.

When the cases that occurred during the first 2 years of follow-up were excluded, the HRs for endometrial and ovarian cancer were slightly higher, whereas the results for breast cancer remained unchanged.

None of the interaction terms between acrylamide intake and covariables was statistically significant.

\section{Discussion}

This prospective cohort study provides the first epidemiologic indications that dietary acrylamide intake may increase the risk of cancer in humans. For both endometrial and ovarian cancer, a positive association with dietary acrylamide was observed, particularly in the subgroup of never-smoking women. Whether the differences between the total group and the neversmokers are due to effect modification by smoking or due to masking of the dietary acrylamide effect by acrylamide or other compounds in tobacco smoke remains to be established. Smoking may induce epoxide hydrolase, which results in enhanced metabolism of glycidamide (33), after which there is less glycidamide left to damage DNA.

There was no association between acrylamide intake and postmenopausal breast cancer, which is in line with the only other prospective cohort study on this topic (23), although this latter study investigated mainly premenopausal breast cancer. According to the formula of Cai et 
al. (39) for calculating power for case-cohort studies, we had a power of $80 \%$ to detect a HR of breast cancer 1.38 or higher for the total group and 1.52 or higher for the never-smokers for comparing the highest quintile of acrylamide intake to the lowest.

The relationship between dietary acrylamide intake and endometrial cancer has not been studied before in humans, but the positive association is in line with an increased occurrence of uterus tumors in rats after acrylamide exposure (9). The only epidemiologic study thus far on acrylamide and ovarian cancer, a case-control study, showed no association (19). This may be due to preclinical disease affecting food consumption in the casecontrol study. Generally speaking, case-control studies may suffer from recall bias, but whether this played a role in this particular case-control study is not clear. Recall bias relating to acrylamide itself is unlikely, since the dietary data were gathered before 2002, when acrylamide in food was first reported. However, recall bias may have occurred for acrylamide-containing foods, such as coffee or French fries. Protopathic bias is not present in our prospective cohort study, in which exclusion of cases in the first 2 years of follow-up did not alter the conclusions. Another reason for the absence of an association in the case-control study might be the non-differential misclassification of the acrylamide intake, which biases the effect estimate toward null. Pelucchi et al. based their acrylamide intake assessment on acrylamide levels of foods that were not all specifically from the country of the study population, and they did not obtain information from the study participants on individual portion sizes, except for fried potatoes.

The acrylamide intake assessment is an important asset of the present study, together with its large study size and prospective nature, which precludes recall bias and makes it less vulnerable to selection. We used acrylamide levels of foods from the Dutch market only and specifically sampled and analyzed foods that were relevant for the NLCS population. Furthermore, the acrylamide measurements were of good quality, which was shown by participation in several international comparison studies of acrylamide analyses $(5,40)$.

Although we have no direct data for acrylamide itself, the reliability and validity of the Netherlands Cohort Study on diet and Cancer (NLCS) Ford Frequency Questionaire (FFQ) for acrylamide can be derived from nutrients that are correlated to acrylamide, namely, carbohydrates and dietary fiber. For carbohydrates, the test-retest correlation was 0.67 for women. For fiber, the corresponding number was 0.69. Furthermore, the decline of the correlation between the baseline questionnaire and the questionnaire administered after 5 years of follow-up was 0.07 on average among the nutrients that were tested. This indicates that although the questionnaire was administered only once, it characterizes longterm food intake for over a period of at least 5 years (30). As for validity, the correlation coefficients between the FFQ and a dietary record method were 0.77 for carbohydrates and 0.74 for fiber. For the food groups potatoes, bread, and cakes and cookies, Spearman correlation coefficients were $0.74,0.80$ and 0.65 , respectively (30).

Acrylamide intake could be difficult to assess due to the large range of acrylamide levels within foods. Thus, there is potential for non-differential misclassifi- cation of acrylamide intake, which would bias risk estimates toward null. We did a validation study to investigate whether using mean acrylamide levels in foods can still result in a sound estimate of total acrylamide intake. ${ }^{4}$ We estimated the acrylamide content of 39 Dutch duplicate 24-h meals that were collected by the Dutch National Institute for Public Health and the Environment in 2004. From the diaries of the participants of the duplicate meal study on the amounts of specific foods that were eaten during the $24 \mathrm{~h}$, the acrylamide content of the 24-h meals was estimated ( $=$ amount of specific food $\times$ mean acrylamide level of a specific food), using the mean acrylamide levels of the specific foods that were also used for the NLCS acrylamide intake assessment. Furthermore, the acrylamide levels of the duplicate meals were chemically analyzed (average \pm SD acrylamide content, $74 \pm 66 \mu \mathrm{g}$ ) and correlated to the estimated acrylamide content (average \pm SD estimated acrylamide content: $107 \pm 74 \mu \mathrm{g}$ ), which rendered a Spearman correlation coefficient of 0.78 . This indicates that it is feasible to make a sound rank ordering of the acrylamide intake via a $24-h$ meal using these mean acrylamide levels for individual foods. For estimating long-term, usual acrylamide intake in the NLCS study, the mean acrylamide levels for foods are expected to be even more suitable because people do not always stick to one particular brand for a certain food, but will switch between brands, at least to some extent. Furthermore, there is also variation in acrylamide levels within batches of the same brand. Over time, differences in acrylamide intake of an individual due to different brands and within-brand fluctuations are, to some extent, averaged out.

This study has a number of limitations. The foods that were sampled and analyzed in 2002 and 2005 may not be completely representative of the foods that were on the market in 1986. Since its discovery in food in 2002, industry and science are working together to reduce acrylamide levels in foods. Nevertheless, it proves to be quite hard to substantially lower the levels (41). Before 2002, some changes in acrylamide levels in foods may have occurred due to changes in production procedures, but never with the intent of lowering acrylamide levels. The changes that may have occurred will have resulted in non-differential misclassification of the intake of our cohort, which will then have led to some underestimation of the true associations between acrylamide and the cancer types we studied.

We did not gather information on whether food was bought or prepared at home and have therefore not taken this into account in the analyses. Of the important acrylamide-containing foods, French fries were most likely to be prepared at home in the NLCS population. However, French fries contribute relatively little to the acrylamide intake and to the variance in acrylamide intake in this cohort. Dutch spiced cake, which is an important acrylamide source in this cohort, was not prepared at home. Besides this, the misclassification that comes from the consumption of home-cooked or

\footnotetext{
${ }^{4}$ Janneke G. Hogervorst, Leo J. Schouten, Erik J. Konings, R. Alexandra Goldbohm, Piet A. van den Brandt, unpublished results.
} 
store-bought foods is probably non-differential and would have biased the risk estimates toward null.

Furthermore, it has to be born in mind that the variation in acrylamide intake in our study was largely due to Dutch spiced cake. Dutch spiced cake resembles gingerbread of the cake-like, non-crusty, type. It does not always contain ginger, but it contains spices and sometimes fruits. Levels of acrylamide in Dutch spiced cake (mean, 1,018 ppb) are much higher than in plain cake (mean, $<30 \mathrm{ppb}$ ). Due to the addition of glucoseand fructose-containing syrup, it contains a higher amount of reducing sugars than other cakes. In the presence of ammonium hydrogen carbonate, which is often used as a baking agent in spiced cake, the formation of acrylamide is further enhanced (42). Our analyses show that it is not just Dutch spiced cake that is responsible for the observed associations because the associations between acrylamide intake and endometrial and ovarian cancer were still present after adjustment for spiced cake, and also in the subgroup of nonconsumers of spiced cake (data for the latter analysis not shown).

The acrylamide-associated HRs that we have observed in this study are considerably higher than the relative risks for dietary acrylamide intake that were calculated based on linear extrapolation of the cancer incidence observed in animal studies (43) and that led some epidemiologists to conclude that epidemiologic studies probably do not have the statistical power to detect an association between dietary acrylamide and cancer. However, the results of extrapolating the high acrylamide dosages that were given to animals to the low dosages that humans are exposed to through food are uncertain. The activity of enzymes in the metabolism of acrylamide may be either higher or lower at low doses, and humans may differ from animals in their ability to handle acrylamide or glycidamide. Furthermore, the genotoxic action of glycidamide $(36,44,45)$ is currently adopted as the mechanism on which acrylamide cancer risk assessments are based. However, the fact that the present study has found associations between acrylamide intake and both endometrial and ovarian cancer might indicate that disturbance of hormonal balances may also be at the basis of acrylamide carcinogenesis (46-48). Acrylamide has been shown to bind to many proteins (49), among which may be proteins related to maintenance of hormonal balances. Park et al. have found a synergistic effect of acrylamide and estrogen on the morphologic transformation of Syrian Hamster Cells (SHE), an end point that is widely used in studies on chemical carcinogenesis (49). This indicates that acrylamide may also be carcinogenic through hormonal pathways, for which animals may not be a good model or for which the linear extrapolation of the animal results to the human situation may not be suitable.

The acrylamide intake of the NLCS population is in the lower part of the WHO estimate range of $0.3-0.8 \mu \mathrm{g} /$ $\mathrm{kg} \mathrm{BW/day} \mathrm{for} \mathrm{developed} \mathrm{countries} \mathrm{(50).} \mathrm{It} \mathrm{corresponds}$ well to the intake level that was obtained from a duplicate meal study in Switzerland, which was 0.28 $\mu \mathrm{g} / \mathrm{kg} \mathrm{BW} /$ day (51), but the authors commented that the participants had consumed far less French fries than the average Swiss population. Current populations, especially young people, consume larger amounts of potato crisps and French fries than the NLCS population did. Because of the high acrylamide levels of these foods, current dietary intake of acrylamide is assumed to be considerably higher $(4,5,52)$ than the intake in this study. On the other hand, industry and science are investigating ways to reduce acrylamide levels in food and have succeeded to some extent for certain foods (53).

If the associations we have observed in this study were found to be causal, acrylamide in food today could prove to be a relevant health hazard. Therefore, we encourage other researchers to prospectively investigate the association between dietary acrylamide intake and cancer in hormone-sensitive organs.

\section{Acknowledgments}

We are indebted to the participants of this study and further wish to thank the cancer registries (IKA, IKL, IKMN, IKN, IKO, IKR, IKST, IKW, IKZ, and VIKC), and the Netherlands nationwide registry of pathology (PALGA). We thank Arnold Kester, Ph.D., from Maastricht University for statistical advice; Sacha van de Crommert, Jolanda Nelissen, Conny de Zwart, and Annemie Pisters from Maastricht University, and Henny Brants, Willy van Dijk, and Martine Jansen from TNO Quality of Life for assistance; Linda van den Bosch from TNO Quality of Life, and Jack Berben and Harry van Montfort from Maastricht University for programming assistance. We are grateful to the Laboratory of Food and Residue Analysis of the Dutch National Institute for Public Health and the Environment for providing the duplicate meals for the validation study.

\section{References}

1. Tareke E, Rydberg P, Karlsson P, Eriksson S, Tornqvist M. Analysis of acrylamide, a carcinogen formed in heated foodstuffs. J Agric Food Chem 2002;50:4998-5006.

2. Summary and conclusions of the sixty-fourth meeting of the Joint FAO/WHO Expert Committee on Food Additives (JECFA): JECFA, 2005.

3. IARC. Monographs on the evaluation of carcinogen risk to humans: some industrial chemicals. Lyon: IARC; 1994.

4. Dybing E, Farmer PB, Andersen M, et al. Human exposure and internal dose assessments of acrylamide in food. Food Chem Toxicol 2005;43:365-410.

5. Konings EJ, Baars AJ, van Klaveren JD, et al. Acrylamide exposure from foods of the Dutch population and an assessment of the consequent risks. Food Chem Toxicol 2003;41:1569-79.

6. Mottram DS, Wedzicha BL, Dodson AT. Acrylamide is formed in the Maillard reaction. Nature 2002;419:448-9.

7. Stadler RH, Blank I, Varga N, et al. Acrylamide from Maillard reaction products. Nature 2002;419:449-50.

8. Taeymans D, Wood J, Ashby P, et al. A review of acrylamide: an industry perspective on research, analysis, formation, and control. Crit Rev Food Sci Nutr 2004;44:323-47.

9. Johnson KA, Gorzinski SJ, Bodner KM, et al. Chronic toxicity and oncogenicity study on acrylamide incorporated in the drinking water of Fischer 344 rats. Toxicol Appl Pharmacol 1986;85:154-68.

10. Bull RJ, Robinson M, Laurie RD, et al. Carcinogenic effects of acrylamide in Sencar and A/J mice. Cancer Res 1984;44:107-11.

11. Bull RJ, Robinson $M$, Stober JA. Carcinogenic activity of acrylamide in the skin and lung of Swiss-ICR mice. Cancer Lett 1984;24:209-12

12. Friedman MA, Dulak LH, Stedham MA. A lifetime oncogenicity study in rats with acrylamide. Fundam Appl Toxicol 1995;27:95-105.

13. Marsh GM, Lucas LJ, Youk AO, Schall LC. Mortality patterns among workers exposed to acrylamide: 1994 follow up. Occup Environ Med 1999;56:181-90.

14. Schulz MR, Hertz-Picciotto I, van Wijngaarden E, Hernandez JC, Ball LM. Dose-response relation between acrylamide and pancreatic cancer. Occup Environ Med 2001;58:609.

15. Sobel W, Bond GG, Parsons TW, Brenner FE. Acrylamide cohort mortality study. Br J Ind Med 1986;43:785-8.

16. Collins JJ, Swaen GM, Marsh GM, Utidjian HM, Caporossi JC, Lucas LJ. Mortality patterns among workers exposed to acrylamide. J Occup Med 1989;31:614-7. 
17. Swaen GM, Haidar S, Burns CJ, et al. Mortality study update of acrylamide workers. Occup Environ Med 2007;64:396-401.

18. Marsh GM, Youk AO, Buchanich JM, Kant IJ, Swaen G. Mortality patterns among workers exposed to acrylamide: updated follow up. J Occup Environ Med 2007;49:82-95.

19. Pelucchi C, Galeone C, Levi F, et al. Dietary acrylamide and human cancer. Int J Cancer 2006;118:467-71.

20. Mucci LA, Dickman PW, Steineck G, Adami HO, Augustsson K. Dietary acrylamide and cancer of the large bowel, kidney, and bladder: absence of an association in a population-based study in Sweden. Br J Cancer 2003;88:84-9.

21. Mucci LA, Lindblad P, Steineck G, Adami HO. Dietary acrylamide and risk of renal cell cancer. Int J Cancer 2004;109:774-6.

22. Mucci LA, Adami HO, Wolk A. Prospective study of dietary acrylamide and risk of colorectal cancer among women. Int J Cancer 2006;118:169-73.

23. Mucci LA, Sandin S, Balter K, Adami HO, Magnusson C, Weiderpass E. Acrylamide intake and breast cancer risk in Swedish women. Jama 2005;293:1326-7.

24. Zhang M, Yang ZY, Binns CW, Lee AH. Diet and ovarian cancer risk: a case-control study in China. Br J Cancer 2002;86:712-7.

25. Michels KB, Rosner BA, Chumlea WC, Colditz GA, Willett WC. Preschool diet and adult risk of breast cancer. Int J Cancer 2006;118: 749-54.

26. van den Brandt PA, Goldbohm RA, van 't Veer P, Volovics A, Hermus RJ, Sturmans F. A large-scale prospective cohort study on diet and cancer in the Netherlands. J Clin Epidemiol 1990;43: 285-95.

27. Goldbohm RA, van den Brandt PA, Dorant E. Estimation of the coverage of Dutch municipalities by cancer registries and PALGA based on hospital discharge data. Tijdschr Soc Gezondheidsz 1994;72: $80-84$.

28. van den Brandt PA, Schouten LJ, Goldbohm RA, Dorant E, Hunen PM. Development of a record linkage protocol for use in the Dutch Cancer Registry for Epidemiological Research. Int J Epidemiol 1990; 19:553-8

29. Goldbohm RA, van den Brandt PA, Brants HA, et al. Validation of a dietary questionnaire used in a large-scale prospective cohort study on diet and cancer. Eur J Clin Nutr 1994;48:253-65.

30. Goldbohm RA, van 't Veer P, van den Brandt PA, et al. Reproducibility of a food frequency questionnaire and stability of dietary habits determined from five annually repeated measurements. Eur J Clin Nutr 1995;49:420-9.

31. Database of the European Commission Institute for Reference Materials and Measurements (IRMM; accessed August 3, 2006, at http://www.irmm.jrc.be/html/activities/acrylamide/database.htm).

32. Schottenfeld D, Fraumeni JF, Jr. Cancer epidemiology and prevention. New York City: Oxford University Press; 2006.

33. Schettgen T, Rossbach B, Kutting B, Letzel S, Drexler H, Angerer J. Determination of haemoglobin adducts of acrylamide and glycidamide in smoking and non-smoking persons of the general population. Int J Hyg Environ Health 2004;207:531-9.

34. Bergmark E. Hemoglobin adducts of acrylamide and acrylonitrile in laboratory workers, smokers and nonsmokers. Chem Res Toxicol 1997;10:78-84.

35. Ardies CM, Smith TJ, Kim S, Yang CS. Induction of 4-(methylnitrosamino)-1-(3-pyridyl)-1-butanone (NNK) activation in rat lung microsomes by chronic ethanol consumption and repeated running exercise. Cancer Lett 1996;103:209-18.

36. Ghanayem BI, Witt KL, Kissling GE, Tice RR, Recio L. Absence of acrylamide-induced genotoxicity in CYP2E1-null mice: evidence consistent with a glycidamide-mediated effect. Mutat Res 2005;578: $284-97$.

37. Howard LA, Micu AL, Sellers EM, Tyndale RF. Low doses of nicotine and ethanol induce CYP2E1 and chlorzoxazone metabolism in rat liver. J Pharmacol Exp Ther 2001;299:542-50.

38. Wang Z, Hall SD, Maya JF, Li L, Asghar A, Gorski JC. Diabetes mellitus increases the in vivo activity of cytochrome P450 2E1 in humans. Br J Clin Pharmacol 2003;55:77-85.

39. Cai J, Zeng D. Sample size/power calculation for case-cohort studies. Biometrics 2004;60:1015-24.

40. Wenzl T, Karasek L, Rosen J, et al. Collaborative trial validation study of two methods, one based on high performance liquid chromatography-tandem mass spectrometry and on gas chromatography-mass spectrometry for the determination of acrylamide in bakery and potato products. J Chromatogr A 2006;1132:211-8.

41. Stadler RH. Acrylamide formation in different foods and potential strategies for reduction. Adv Exp Med Biol 2005;561:157-69.

42. Amrein TM, Andres L, Manzardo GG, Amado R. Investigations on the promoting effect of ammonium hydrogen carbonate on the formation of acrylamide in model systems. J Agric Food Chem 2006; 54:10253-61.

43. Dybing E, Sanner T. Risk assessment of acrylamide in foods. Toxicol Sci 2003;75:7-15.

44. Gamboa da Costa G, Churchwell MI, Hamilton LP, et al. DNA adduct formation from acrylamide via conversion to glycidamide in adult and neonatal mice. Chem Res Toxicol 2003;16:1328-37.

45. Doerge DR, da Costa GG, McDaniel LP, Churchwell MI, Twaddle NC, Beland FA. DNA adducts derived from administration of acrylamide and glycidamide to mice and rats. Mutat Res 2005;580: $131-41$.

46. Akhmedkhanov A, Zeleniuch-Jacquotte A, Toniolo P. Role of exogenous and endogenous hormones in endometrial cancer: review of the evidence and research perspectives. Ann N Y Acad Sci 2001; 943:296-315

47. Cramer DW, Welch WR. Determinants of ovarian cancer risk. II. Inferences regarding pathogenesis. J Natl Cancer Inst 1983;71: $717-21$.

48. Exon JH. A review of the toxicology of acrylamide. J Toxicol Environ Health B Crit Rev 2006;9:397-412.

49. Park J, Kamendulis LM, Friedman MA, Klaunig JE. Acrylamideinduced cellular transformation. Toxicol Sci 2002;65:177-83.

50. Health implications of acrylamide in food. Report of a Joint FAO/ WHO Consultation.: FAO/WHO; 2002

51. Swiss Federal Office of Public Health. Assessment of acrylamide intake by duplicate diet study. 2002. (accessed August 3, 2006, at http://www.bag.admin.ch).

52. Matthys C, Bilau M, Govaert Y, Moons E, De Henauw S, Willems JL. Risk assessment of dietary acrylamide intake in Flemish adolescents. Food Chem Toxicol 2005;43:271-8.

53. Acrylamide Status Report December 2004: a summary of the efforts and progress achieved to date by the European food and drink industry (CIAA) in lowering levels of acrylamide in food. Bruxelles: CIAA; 2004 